\title{
Comparison of PANAMutyper and PNAClamp for Detecting KRAS Mutations from Patients With Malignant Pleural Effusion
}

\author{
SU YEON CHOI ${ }^{1,2^{*}}$, HYUNG WOO KIM ${ }^{1}$, SANG HOON JEON ${ }^{1}$, BIT NA KIM ${ }^{1,2}$, NAHYEON KANG ${ }^{1,2}$, \\ CHANG DONG YEO ${ }^{1,2}$, CHAN KWON PARK ${ }^{1,2}$, YOUNG KYOON KIM ${ }^{1}$, YOON HO LEE ${ }^{3}, \mathrm{KYO} \mathrm{YOUNG} \mathrm{LEE}^{3}$, \\ SUG HYUNG LEE ${ }^{4}$, JONG Y. PARK ${ }^{5}$, MI SUN PARK ${ }^{6}$, HYEON WOO YIM ${ }^{6}$ and SEUNG JOON KIM ${ }^{1,2}$ \\ ${ }^{1}$ Division of Pulmonology, Department of Internal Medicine, College of Medicine, \\ The Catholic University of Korea, Seoul, Republic of Korea; \\ ${ }^{2}$ The Cancer Research Institute, College of Medicine, The Catholic University of Korea, Seoul, Republic of Korea; \\ ${ }^{3}$ Department of Hospital Pathology, College of Medicine, \\ The Catholic University of Korea, Seoul, Republic of Korea; \\ ${ }^{4}$ Department of Pathology, College of Medicine, The Catholic University of Korea, Seoul, Republic of Korea; \\ ${ }^{5}$ Department of Cancer Epidemiology, Moffitt Cancer Center, Tampa, FL, U.S.A.; \\ ${ }^{6}$ Department of Biostatistics, Clinical Research Coordinating Center, \\ The Catholic University of Korea, Seoul, Republic of Korea
}

\begin{abstract}
Background/Aim: KRAS is one of the frequently mutated genes in human cancers and often relates with drug resistance and poor prognosis. PANAMutyper ${ }^{T M}$ is a novel technology that integrates PNAClamp ${ }^{T M}$ and PANA S-Melting ${ }^{T M}$. In the present study, PANAMutyper ${ }^{T M}$ and PNAClamp ${ }^{T M}$ were compared for the detection of KRAS mutations using different samples of patients with malignant pleural effusion. Patients and Methods: A total of 103 patients (including 56 lung adenocarcinoma, 10 lung squamous carcinoma, 17 small cell lung cancer, 3 large cell lung cancer, 3 stomach cancer, 2 ovarian cancer, and others) with malignant pleural effusion were investigated using matched tumor tissue, cell block, and pleural effusion samples. The diagnostic performance of these two methods was compared. Results: KRAS mutations were detected in 18 (17.5\%) of 103 patients using tissue, cell block, and pleural effusion samples. All 18 patients with KRAS mutations were detected by PANAMutyper ${ }^{T M}$ using any sample type, however, only 7 cases
\end{abstract}

This article is freely accessible online.

Correspondence to: Seung Joon Kim, Division of Pulmonology, Department of Internal Medicine, Seoul St. Mary's Hospital, College of Medicine, The Catholic University of Korea, 222, Banpo-daero, Seocho-gu, Seoul, 06591, Republic of Korea; Tel: +82 222586063, Fax: +82 25993589, e-mail: cmcksj@catholic.ac.kr

Key Words: PANAMutyper, PNAClamp, KRAS mutation, malignant pleural effusion. were detected by PNAClamp ${ }^{T M}$. Among the subtypes of KRAS mutations, substitution in codon 12, 35G>T was the most frequent, followed by substitution in codon 12, 35G $>A$ and codon 12, 34G>A. In pleural effusion specimens, PANAMutyper ${ }^{T M}$ showed a better diagnostic performance compared to PNAClamp ${ }^{T M}$. Conclusion: PANAMutyper ${ }^{T M}$ had a diagnostic superiority for the detection of KRAS mutations in patients with malignant pleural effusion compared to PNAClamp ${ }^{T M}$, although there was a concordance between PANAMutyper ${ }^{T M}$ and PNAClamp ${ }^{T M}$ results. Therefore, PANAMutyper ${ }^{T M}$ can be used for a more sensitive and accurate detection of KRAS mutations.

KRAS is a well-known signaling molecule in the epidermal growth factor receptor pathway. For many years, KRAS has been recognized as one of the most frequently mutated oncogenes in multiple human cancers, including pancreatic, colorectal, lung, endometrial, gastric, biliary tract, and thyroid cancer $(1,2)$. The existence of KRAS mutations is commonly associated with poor prognosis and resistance to therapy (3-7).

Previously, we compared PNA clamping with direct sequencing for the detection of KRAS and EGFR mutations and found that the diagnostic performance and clinical outcome using PNA clamping are better compared to those of direct sequencing $(8,9)$. PNAClamp ${ }^{\mathrm{TM}}$ technology is based on the principle that PNA can inhibit amplification of wild-type DNA by hybridizing with wild-type sequences. Therefore, mutant DNA is preferentially amplified, and this is detected by an intercalating dye (10-13). 
PANAMutyper ${ }^{\mathrm{TM}}$ R KRAS is a novel kit based on PANA C-Melting ${ }^{\mathrm{TM}}$ technology, combining PNAClamp ${ }^{\mathrm{TM}}$ and PANA S-Melting ${ }^{\mathrm{TM}}$, a multiplex detection system using specific PNA detection probes. Similar to PNAClamp ${ }^{\mathrm{TM}}$, a PNA clamp probe in PANAMutyper ${ }^{\mathrm{TM}}$ can only tightly bind to wild-type DNA sequences, and thus suppresses their amplification during PCR. Meanwhile, the PNA detection probe in PANAMutyper ${ }^{\mathrm{TM}}$ is conjugated with a fluorescent dye and a quencher and can specifically detect target mutant DNA. Each mutation can be genotyped by melting peak analysis $(14,15)$.

In the present study, we analyzed KRAS mutations in matched tumor tissues, cell blocks, and pleural effusion samples by PANAMutyper ${ }^{\mathrm{TM}}$ (both clamping-assisted fluorescence and melting curve analysis) and PNAClamp ${ }^{\mathrm{TM}}$ (clamping only), to compare their diagnostic performance and determine their usefulness in detecting KRAS mutations. To the best of our knowledge, this is the first study that uses PNA clamping-assisted fluorescence melting curve analysis to detect KRAS mutations in matched tissue, cell block, and pleural effusion samples to compare its performance with PNA clamping only.

\section{Patients and Methods}

Study subjects. We investigated 103 patients with malignant pleural effusion who underwent diagnostic thoracentesis at the Division of Pulmonology of Seoul St. Mary's Hospital (Seoul, Republic of Korea), between September 2008 and December 2016. We used samples of malignant pleural effusion diagnosed after confirming the presence of malignant cells using cytological examination, pleural biopsy or histology without other cause of pleural effusion. Pleural fluid samples from all patients were evaluated for KRAS mutations using both PANAMutyper ${ }^{\mathrm{TM}}$ and PNAClamp ${ }^{\mathrm{TM}}$. For patients whose tumor tissue and cell block samples were available, KRAS mutations were investigated from these as well.

All subjects provided a written informed consent for the procedure, and the study protocol was approved by the Institutional Review Board of Seoul St. Mary's Hospital, The Catholic University of Korea (IRB approval number: KC16TISI0672).

DNA extraction. 5- $\mu \mathrm{m}$ paraffin sections of tissues and cell blocks were used for DNA extraction. These sections were deparaffinized in xylene and were washed in ethanol. Five $\mathrm{ml}$ of pleural fluid specimens were centrifuged, and $1 \mathrm{ml}$ of the supernatant was used for DNA analysis. DNA was extracted using a High Pure PCR Template Preparation Kit (Roche Applied Science, Mannheim, Germany). After eluting DNA in $50 \mu \mathrm{l}$ of elution buffer, concentration and purity of extracted DNA were evaluated using a NanoDrop ND-1000 spectrophotometer (NanoDrop Technologies, Wilmington, DE, USA). The amount of DNA used was 35-70 ng (5-10 ng/reaction) for PNAClamp $^{\mathrm{TM}}$ (version 4), and 40-80 ng (5-10 ng/reaction) for PANAMutyper ${ }^{\mathrm{TM}}$. A schematic representation of the workflow for PNAClamp $^{\mathrm{TM}}$ versus $^{\text {PANAMutyper }}{ }^{\mathrm{TM}}$ is presented in Figure 1.

PNAClamp $^{T M}$. PNAClamp ${ }^{\mathrm{TM}}$ analysis was performed using the PNAClamp ${ }^{\mathrm{TM}}$ KRAS Mutation Detection Kit (Panagene, Daejeon, Republic of Korea), following the manufacturer's instructions.
Table I. Clinical characteristics of patients.

\begin{tabular}{lcc}
\hline Variables & $\mathrm{n}$ & $\%$ \\
\hline Gender & & \\
Male & 65 & 63.1 \\
Female & 38 & 36.9 \\
Age (years) & & \\
Mean \pm standard deviation & $72 \pm 12$ & \\
Median (range) & $74(31-93)$ & \\
Smoking status & & \\
Never smoker & 52 & 50.5 \\
Ex-smoker & 38 & 36.9 \\
Current smoker & 13 & 12.6 \\
Pathology & & \\
Adenocarcinoma & 56 & 54.4 \\
Squamous cell carcinoma & 10 & 9.7 \\
Small cell carcinoma & 17 & 16.5 \\
Large cell carcinoma & 3 & 2.9 \\
Sarcomatoid carcinoma & 1 & 1.0 \\
NSCLC NOS & 2 & 1.9 \\
Stomach cancer & 3 & 2.9 \\
Mesothelioma & 2 & 1.9 \\
Ovarian cancer & 2 & 1.9 \\
Thyroid cancer & 2 & 1.9 \\
Breast cancer & 1 & 1.0 \\
Endometrial cancer & 1 & 1.0 \\
Esophageal cancer & 1 & 1.0 \\
Head and neck cancer & 1 & \\
Lymphoma & 1 & \\
Total & & \\
\hline & & \\
\hline
\end{tabular}

NSCLC NOS: Non-small cell lung cancer not otherwise specified.

Briefly, $7 \mu \mathrm{l}$ of DNA template, $3 \mu \mathrm{l}$ of each PNA mix, and $10 \mu \mathrm{l}$ of $2 \mathrm{X}$ premix were mixed for a single amplification reaction. Amplification of the mixture $(20 \mu \mathrm{l})$ was performed in a CFX96 real-time PCR instrument (Bio-Rad Laboratories, CA, USA) with the following thermal program: pre-incubation at $94^{\circ} \mathrm{C}$ for $5 \mathrm{~min}$, followed by 40 cycles of amplification at $94^{\circ} \mathrm{C}$ for $30 \mathrm{sec}(\mathrm{s}), 70^{\circ} \mathrm{C}$ for $30 \mathrm{~s}, 63^{\circ} \mathrm{C}$ for $30 \mathrm{~s}$, and $72^{\circ} \mathrm{C}$ for $30 \mathrm{~s}$.

Detection of signal from the intercalating dye was measured at every $63^{\circ} \mathrm{C}$ step. Cycle threshold $(\mathrm{Ct}$ ) value for the reaction (sample $\mathrm{Ct}$ value) was determined based on the fluorescence value measured at every $63^{\circ} \mathrm{C}$ step. Results were assessed according to the delta-Ct value, calculated by subtracting the $\mathrm{Ct}$ value of the sample from the $\mathrm{Ct}$ value of the standard. Delta-Ct values larger than 2.0 were interpreted as corresponding to mutant DNA.

PANAMutyper ${ }^{T M}$. PANAMutyper ${ }^{\mathrm{TM}}$ analysis was performed using the PANAMutyper ${ }^{\mathrm{TM}}$ R KRAS kit (Panagene, Deajeon, Republic of Korea), following the manual provided by the manufacturer. Briefly, $5 \mu \mathrm{l}$ of DNA template, $19 \mu \mathrm{l}$ of each master mix, and $1 \mu \mathrm{l}$ of Taq polymerase were mixed for a single amplification reaction. Amplification of the mixture $(25 \mu \mathrm{l})$ was performed in a CFX96 real-time PCR instrument (Bio-Rad) using the following thermal program: a UDG incubation at $50^{\circ} \mathrm{C}$ for $2 \mathrm{~min}$, a pre-incubation at $95^{\circ} \mathrm{C}$ for $15 \mathrm{~min}$, first amplification with 15 cycles of $95^{\circ} \mathrm{C}$ for 30 $\mathrm{s}, 70^{\circ} \mathrm{C}$ for $20 \mathrm{~s}$, and $63^{\circ} \mathrm{C}$ for $1 \mathrm{~min}$, a second amplification with 35 cycles of $95^{\circ} \mathrm{C}$ for $10 \mathrm{~s}, 53^{\circ} \mathrm{C}$ for $20 \mathrm{~s}$, and $73^{\circ} \mathrm{C}$ for $20 \mathrm{~s}$, a 
A

Tissue, cell block, pleural effusion



Input diluted DNA sample and reagent mix in a 96-well plate

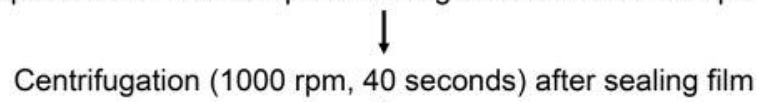

$\downarrow$

Input 96-well plate and run instrument

$\downarrow$

PNAClamp ${ }^{\mathrm{TM}}$ running time - about 1 hour 40 minutes

PANAMutyper ${ }^{\mathrm{TM}}$ running time - about 2 hours 40 minutes

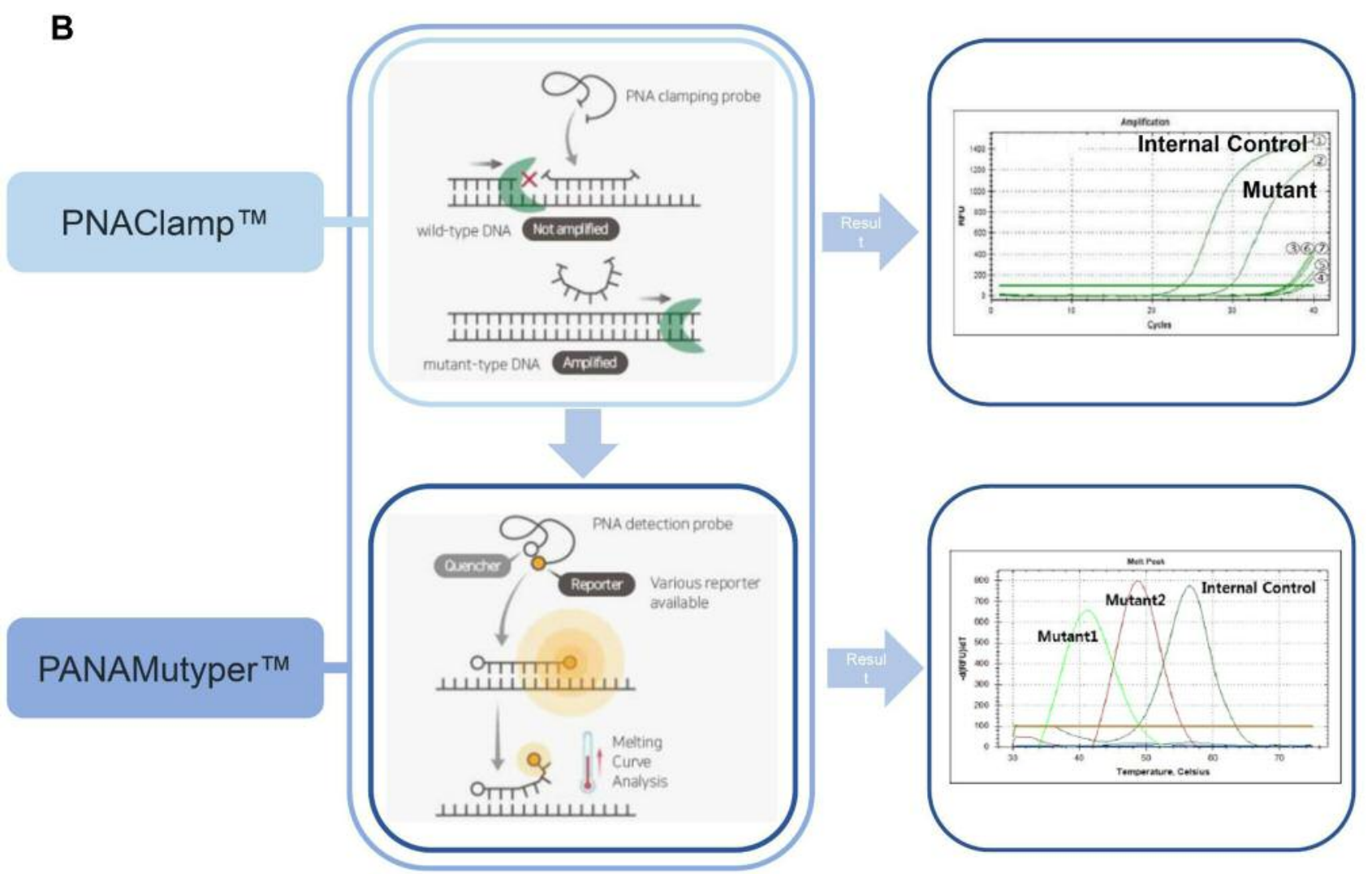

Figure 1. Schematic representation of the workflow for PNAClamp ${ }^{T M} v s$ PANAMutyper ${ }^{T M}$. (A) Experimental methods for DNA extraction and the performance of PNAClamp ${ }^{T M}$ and PANAMutyper ${ }^{T M}$. (B) PANAMutyper ${ }^{T M}$ is a fusion technology of PNAClamp ${ }^{T M}$ for high sensitivity and PCR for multiplex genotyping. *Adapted from the PANAGENE INC. (http://www.panagene.com/_ENG/html/dh/tech2).

product denaturation at $95^{\circ} \mathrm{C}$ for $15 \mathrm{~min}$, a detection probe binding at $35^{\circ} \mathrm{C}$ for $5 \mathrm{~min}$, and a melting curve analysis at $35^{\circ} \mathrm{C}$ to $75^{\circ} \mathrm{C}$ with an increment of $0.5^{\circ} \mathrm{C}$ and detection for $3 \mathrm{~s}$. Four color fluorescence signals (FAM, HEX, ROX, and Cy5) were measured during the melting curve analysis. Following amplification and melting analysis, the genotype of each sample was determined by the melting temperature (Tm) value obtained from the melting curve of each fluorescent dye. Each sample was assessed according to specific fluorescence and $\mathrm{Tm}$ ranges provided by the manual. 
Table II. Distribution of KRAS mutations in 103 patients with malignant effusion.

\begin{tabular}{|c|c|c|c|c|c|c|c|c|c|c|}
\hline \multirow{3}{*}{ No. } & \multirow{3}{*}{ Gender } & \multirow{3}{*}{ r Age } & \multirow{3}{*}{$\begin{array}{l}\text { Smoking } \\
\text { status }\end{array}$} & \multirow{3}{*}{$\begin{array}{l}\text { Underlying } \\
\text { malignancy }\end{array}$} & \multicolumn{6}{|c|}{ Samples and methods for the detection of KRAS mutations } \\
\hline & & & & & \multicolumn{2}{|c|}{ Tissue } & \multicolumn{2}{|c|}{ Cell block } & \multicolumn{2}{|c|}{ Effusion } \\
\hline & & & & & PANAMutyper ${ }^{\mathrm{TM}}$ & PNAClamp ${ }^{\mathrm{TM}}$ & PANAMutyper ${ }^{\mathrm{TM}}$ & PNAClamp ${ }^{\mathrm{TM}}$ & PANAMutyper ${ }^{\mathrm{TM}}$ & PNAClamp ${ }^{\mathrm{TM}}$ \\
\hline 1 & M & 69 & Current & $\mathrm{ADC}$ & Ind. & Ind. & Codon $1235 \mathrm{G}>\mathrm{T}$ & WT & WT & WT \\
\hline 2 & M & 57 & Current & $\mathrm{ADC}$ & NA & NA & NA & NA & Codon $1234 \mathrm{G}>\mathrm{A}$ & WT \\
\hline 3 & $\mathrm{~F}$ & 59 & Never & $\mathrm{ADC}$ & NA & NA & Codon 12 35G $>\mathrm{A}$ & WT & Codon $1235 \mathrm{G}>\mathrm{A}$ & WT \\
\hline 4 & M & 74 & Never & $\mathrm{ADC}$ & NA & NA & Codon $61182 \mathrm{~A}>\mathrm{T}$ & WT & WT & WT \\
\hline 5 & M & 74 & Never & $\mathrm{ADC}$ & NA & NA & Codon $61182 \mathrm{~A}>\mathrm{T}$ & WT & WT & WT \\
\hline 6 & M & 92 & Ex & $\mathrm{ADC}$ & NA & NA & Codon $1235 \mathrm{G}>\mathrm{C}$ & WT & WT & WT \\
\hline 7 & M & 86 & Ex & $\mathrm{ADC}$ & NA & NA & NA & NA & Codon $1234 \mathrm{G}>\mathrm{A}$ & WT \\
\hline 8 & M & 61 & Current & $\mathrm{ADC}$ & Codon $1338 \mathrm{G}>\mathrm{C}$ & WT & WT & WT & WT & WT \\
\hline 9 & $\mathrm{~F}$ & 71 & Never & $\mathrm{ADC}$ & WT & WT & NA & NA & Codon 13 37G $>$ A & WT \\
\hline 10 & M & 77 & Ex & $\mathrm{ADC}$ & Codon $1235 \mathrm{G}>\mathrm{T}$ & WT & NA & NA & Codon $1235 \mathrm{G}>\mathrm{T}$ & Codon 12 \\
\hline 11 & $\mathrm{~F}$ & 31 & Never & $\mathrm{ADC}$ & NA & NA & NA & NA & Codon $1235 \mathrm{G}>\mathrm{A}$ & WT \\
\hline 12 & $\mathrm{~F}$ & 79 & Never & $\mathrm{ADC}$ & NA & NA & NA & NA & Codon $1235 \mathrm{G}>\mathrm{A}$ & Codon 12 \\
\hline 13 & $\mathrm{~F}$ & 79 & Never & $\mathrm{ADC}$ & Codon 13 37G $>$ A & WT & WT & WT & WT & WT \\
\hline 14 & $\mathrm{~F}$ & 62 & Never & $\mathrm{ADC}$ & NA & NA & NA & NA & WT & WT \\
\hline 15 & M & 68 & Ex & $\mathrm{ADC}$ & NA & NA & WT & WT & WT & WT \\
\hline 16 & M & 74 & Never & $\mathrm{ADC}$ & NA & NA & NA & NA & WT & WT \\
\hline 17 & M & 68 & Never & $\mathrm{ADC}$ & NA & NA & WT & WT & WT & WT \\
\hline 18 & M & 76 & Never & $\mathrm{ADC}$ & WT & WT & NA & NA & WT & WT \\
\hline 19 & M & 65 & Ex & $\mathrm{ADC}$ & WT & WT & NA & NA & WT & WT \\
\hline 20 & M & 74 & Never & $\mathrm{ADC}$ & WT & WT & NA & NA & WT & WT \\
\hline 21 & M & 73 & Never & $\mathrm{ADC}$ & NA & NA & WT & WT & WT & WT \\
\hline 22 & M & 47 & Never & $\mathrm{ADC}$ & NA & NA & WT & WT & WT & WT \\
\hline 23 & M & 71 & Never & $\mathrm{ADC}$ & NA & NA & NA & NA & WT & WT \\
\hline 24 & M & 52 & Ex & $\mathrm{ADC}$ & NA & NA & WT & WT & WT & WT \\
\hline 25 & $\mathrm{~F}$ & 76 & Never & $\mathrm{ADC}$ & WT & WT & WT & WT & WT & WT \\
\hline 26 & $\mathrm{~F}$ & 83 & Never & $\mathrm{ADC}$ & WT & WT & WT & WT & WT & WT \\
\hline 27 & $\mathrm{~F}$ & 48 & Ex & $\mathrm{ADC}$ & WT & WT & WT & WT & WT & WT \\
\hline 28 & M & 60 & Ex & $\mathrm{ADC}$ & NA & NA & WT & WT & WT & WT \\
\hline 29 & $\mathrm{~F}$ & 60 & Never & $\mathrm{ADC}$ & WT & WT & WT & WT & WT & WT \\
\hline 30 & $\mathrm{M}$ & 73 & Ex & $\mathrm{ADC}$ & WT & WT & NA & NA & WT & WT \\
\hline 31 & $\mathrm{~F}$ & 88 & Never & $\mathrm{ADC}$ & WT & WT & WT & WT & WT & WT \\
\hline 32 & M & 77 & Ex & $\mathrm{ADC}$ & NA & NA & NA & NA & WT & WT \\
\hline 33 & $\mathrm{~F}$ & 86 & Never & $\mathrm{ADC}$ & WT & WT & WT & WT & WT & Ind. \\
\hline 34 & $\mathrm{M}$ & 89 & Ex & $\mathrm{ADC}$ & NA & NA & NA & NA & WT & WT \\
\hline 35 & $\mathrm{~F}$ & 80 & Never & $\mathrm{ADC}$ & NA & NA & NA & NA & WT & WT \\
\hline 36 & $\mathrm{~F}$ & 64 & Never & $\mathrm{ADC}$ & WT & WT & NA & NA & WT & WT \\
\hline 37 & M & 57 & Ex & $\mathrm{ADC}$ & WT & WT & WT & WT & WT & WT \\
\hline 38 & $\mathrm{M}$ & 78 & Ex & $\mathrm{ADC}$ & WT & WT & NA & NA & WT & WT \\
\hline 39 & M & 59 & Ex & $\mathrm{ADC}$ & NA & NA & WT & WT & WT & WT \\
\hline 40 & $\mathrm{~F}$ & 88 & Never & $\mathrm{ADC}$ & NA & NA & WT & WT & WT & WT \\
\hline 41 & $\mathrm{~F}$ & 62 & Never & $\mathrm{ADC}$ & NA & NA & WT & WT & WT & WT \\
\hline 42 & $\mathrm{~F}$ & 62 & Never & $\mathrm{ADC}$ & NA & NA & WT & WT & WT & WT \\
\hline 43 & $\mathrm{~F}$ & 84 & Never & $\mathrm{ADC}$ & WT & WT & NA & NA & WT & WT \\
\hline 44 & M & 76 & Never & $\mathrm{ADC}$ & WT & WT & NA & NA & WT & WT \\
\hline 45 & M & 72 & Ex & $\mathrm{ADC}$ & NA & NA & WT & WT & WT & WT \\
\hline 46 & $\mathrm{~F}$ & 69 & Never & $\mathrm{ADC}$ & NA & NA & WT & WT & WT & WT \\
\hline 47 & $\mathrm{~F}$ & 84 & Never & $\mathrm{ADC}$ & NA & NA & WT & WT & WT & WT \\
\hline 48 & M & 78 & Current & $\mathrm{ADC}$ & WT & WT & WT & WT & WT & WT \\
\hline 49 & M & 68 & Never & $\mathrm{ADC}$ & NA & NA & WT & WT & WT & WT \\
\hline 50 & M & 72 & Ex & $\mathrm{ADC}$ & NA & NA & WT & WT & WT & WT \\
\hline 51 & M & 86 & Ex & $\mathrm{ADC}$ & NA & NA & WT & WT & WT & WT \\
\hline 52 & M & 61 & Ex & $\mathrm{ADC}$ & NA & NA & WT & WT & WT & WT \\
\hline 53 & $\mathrm{~F}$ & 49 & Never & $\mathrm{ADC}$ & NA & NA & NA & NA & WT & WT \\
\hline 54 & $\mathrm{~F}$ & 65 & Never & $\mathrm{ADC}$ & NA & NA & NA & NA & WT & WT \\
\hline
\end{tabular}


Samples and methods for the detection of KRAS mutations

\begin{tabular}{ccccc}
\cline { 3 - 4 } No. Gender Age Smoking & Underlying & Tissue & Cell block & Effusion \\
status & malignancy & &
\end{tabular}

PANAMutyper $^{\mathrm{TM}}$ PNAClamp $^{\mathrm{TM}} \quad$ PANAMutyper $^{\mathrm{TM}}$ PNAClamp $^{\mathrm{TM}}$ PANAMutyper $^{\mathrm{TM}} \quad$ PNAClamp $^{\mathrm{TM}}$

\begin{tabular}{|c|c|c|c|c|c|c|c|c|c|c|}
\hline 55 & $\mathrm{~F}$ & 81 & Never & $\mathrm{ADC}$ & NA & NA & NA & NA & WT & WT \\
\hline 56 & $\mathrm{M}$ & 76 & Current & $\mathrm{ADC}$ & NA & NA & NA & NA & WT & WT \\
\hline 57 & $\mathrm{M}$ & 71 & Ex & SQCC & WT & WT & NA & NA & WT & WT \\
\hline 58 & $\mathrm{~F}$ & 55 & Never & SQCC & NA & NA & WT & WT & WT & WT \\
\hline 59 & M & 82 & Never & SQCC & Ind. & Ind. & NA & NA & WT & WT \\
\hline 60 & $\mathrm{M}$ & 68 & Never & SQCC & NA & NA & WT & WT & WT & WT \\
\hline 61 & $\mathrm{M}$ & 76 & Current & SQCC & WT & WT & NA & NA & WT & WT \\
\hline 62 & M & 87 & Ex & SQCC & WT & WT & NA & NA & WT & WT \\
\hline 63 & $\mathrm{M}$ & 62 & Ex & SQCC & NA & NA & NA & NA & WT & WT \\
\hline 64 & $\mathrm{M}$ & 79 & Ex & SQCC & WT & WT & WT & WT & WT & WT \\
\hline 65 & $\mathrm{M}$ & 81 & Ex & SQCC & NA & NA & NA & NA & WT & WT \\
\hline 66 & $\mathrm{M}$ & 58 & Ex & SQCC & NA & NA & NA & NA & WT & WT \\
\hline 67 & $\mathrm{M}$ & 72 & Ex & SCLC & NA & NA & NA & NA & WT & WT \\
\hline 68 & M & 82 & Never & SCLC & NA & NA & NA & NA & WT & WT \\
\hline 69 & $\mathrm{M}$ & 84 & Ex & SCLC & WT & WT & NA & NA & WT & WT \\
\hline 70 & $\mathrm{M}$ & 77 & Ex & SCLC & WT & WT & WT & WT & WT & WT \\
\hline 71 & $\mathrm{~F}$ & 64 & Never & SCLC & NA & NA & NA & NA & WT & WT \\
\hline 72 & $\mathrm{M}$ & 68 & Ex & SCLC & WT & WT & WT & WT & WT & WT \\
\hline 73 & $\mathrm{M}$ & 70 & Current & SCLC & NA & NA & WT & WT & WT & WT \\
\hline 74 & $\mathrm{M}$ & 79 & Never & SCLC & WT & WT & NA & NA & WT & WT \\
\hline 75 & $\mathrm{~F}$ & 79 & Ex & SCLC & WT & WT & WT & WT & WT & WT \\
\hline 76 & $\mathrm{~F}$ & 87 & Never & SCLC & WT & WT & WT & WT & WT & WT \\
\hline 77 & $\mathrm{M}$ & 69 & Ex & SCLC & WT & WT & NA & NA & WT & WT \\
\hline 78 & $\mathrm{M}$ & 93 & Ex & SCLC & NA & NA & WT & WT & WT & WT \\
\hline 79 & $\mathrm{M}$ & 69 & Current & SCLC & NA & NA & WT & WT & WT & WT \\
\hline 80 & $\mathrm{M}$ & 62 & Current & SCLC & WT & WT & NA & NA & WT & WT \\
\hline 81 & $\mathrm{M}$ & 74 & Current & SCLC & NA & NA & NA & NA & WT & WT \\
\hline 82 & $\mathrm{M}$ & 81 & Ex & SCLC & WT & WT & NA & NA & WT & WT \\
\hline 83 & $\mathrm{~F}$ & 74 & Never & SCLC & WT & WT & WT & WT & WT & WT \\
\hline 84 & $\mathrm{M}$ & 78 & Current & Large cell & WT & WT & NA & NA & Codon $1235 \mathrm{G}>\mathrm{T}$ & Codon 12 \\
\hline 85 & $\mathrm{M}$ & 80 & Ex & Large cell & WT & WT & NA & NA & WT & WT \\
\hline 86 & $\mathrm{M}$ & 64 & Ex & Large cell & WT & WT & NA & NA & WT & WT \\
\hline 87 & M & 79 & Ex & $\begin{array}{l}\text { Sarcomatoid } \\
\text { carcinoma }\end{array}$ & WT & WT & NA & NA & WT & WT \\
\hline 88 & $\mathrm{M}$ & 79 & Current & NSCLC NOS & NA & NA & Codon 12 35G $>\mathrm{T}$ & Codon 12 & Codon $1235 \mathrm{G}>\mathrm{T}$ & WT \\
\hline 89 & $\mathrm{M}$ & 90 & Ex & NSCLC NOS & NA & NA & WT & WT & WT & WT \\
\hline 90 & M & 54 & Current & Stomach cancer & NA & NA & Codon 13 38G $>$ A & WT & Codon $1338 \mathrm{G}>\mathrm{A}$ & Codon 13 \\
\hline 91 & $\mathrm{M}$ & 87 & Never & Stomach cancer & WT & WT & NA & NA & WT & WT \\
\hline 92 & $\mathrm{~F}$ & 85 & Never & Stomach cancer & NA & NA & NA & NA & WT & WT \\
\hline 93 & $\mathrm{~F}$ & 62 & Never & Mesothelioma & NA & NA & NA & NA & WT & WT \\
\hline 94 & $\mathrm{~F}$ & 64 & Never & Mesothelioma & NA & NA & NA & NA & WT & WT \\
\hline 95 & $\mathrm{~F}$ & 84 & Never & Ovarian cancer & NA & NA & Codon 12 34G $>C$ & Codon 12 & Codon $1234 \mathrm{G}>\mathrm{C}$ & Codon 12 \\
\hline 96 & $\mathrm{~F}$ & 49 & Never & Ovarian cancer & WT & WT & Codon $1338 \mathrm{G}>\mathrm{A}$ & Codon 13 & WT & WT \\
\hline 97 & $\mathrm{~F}$ & 74 & Never & Thyroid cancer & NA & NA & WT & WT & WT & WT \\
\hline 98 & $\mathrm{M}$ & 83 & Ex & Thyroid cancer & NA & NA & WT & WT & WT & WT \\
\hline 99 & $\mathrm{~F}$ & 62 & Never & Breast cancer & NA & NA & WT & WT & WT & WT \\
\hline 100 & $\mathrm{~F}$ & 54 & Never & $\begin{array}{l}\text { Endometrial } \\
\text { cancer }\end{array}$ & NA & NA & NA & NA & WT & WT \\
\hline 101 & M & 75 & Ex & $\begin{array}{l}\text { Esophageal } \\
\text { cancer }\end{array}$ & $\mathrm{NA}$ & NA & NA & NA & WT & WT \\
\hline 102 & $\mathrm{~F}$ & 70 & Never & $\begin{array}{c}\text { Head \& } \\
\text { neck cancer }\end{array}$ & WT & WT & NA & NA & WT & WT \\
\hline 103 & $\mathrm{~F}$ & 84 & Never & Lymphoma & WT & WT & NA & NA & WT & WT \\
\hline
\end{tabular}

ADC: Adenocarcinoma; Current: current smoker; Ex: ex-smoker; Ind: indeterminate; NA: not available; Never: never smoker; NSCLC NOS: Nonsmall cell lung cancer not otherwise specified; SQCC: squamous cell carcinoma; SCLC: small cell lung cancer; WT: wild type. 
in vivo $33: 945-954$ (2019)

Table III. Distribution of KRAS mutations detected by PANAMutyper ${ }^{T M}$ and PNAClamp ${ }^{T M}$.

\begin{tabular}{|c|c|c|c|c|c|c|}
\hline & \multicolumn{2}{|c|}{ Tissue } & \multicolumn{2}{|c|}{ Cell block } & \multicolumn{2}{|c|}{ Effusion } \\
\hline & PANAMutyper ${ }^{\mathrm{TM}}$ & PNAClamp ${ }^{\mathrm{TM}}$ & PANAMutyper ${ }^{\mathrm{TM}}$ & PNAClamp ${ }^{\mathrm{TM}}$ & PANAMutyper ${ }^{\mathrm{TM}}$ & PNAClamp $^{\mathrm{TM}}$ \\
\hline Mutation & $3(6.8)$ & - & $9(17.6)$ & $3(5.9)$ & $11(10.7)$ & $5(4.9)$ \\
\hline Codon 12 & - & - & - & $2(3.9)$ & - & $4(3.9)$ \\
\hline Codon $1234 \mathrm{G}>\mathrm{C}$ & - & - & $1(2.0)$ & - & $1(1.0)$ & - \\
\hline Codon 12 34G $>$ A & - & - & - & - & $2(1.9)$ & - \\
\hline Codon $1235 \mathrm{G}>\mathrm{T}$ & $1(2.3)$ & - & $2(3.9)$ & - & $3(2.9)$ & - \\
\hline Codon $1235 \mathrm{G}>\mathrm{A}$ & - & - & $1(2.0)$ & - & $3(2.9)$ & - \\
\hline Codon $1235 \mathrm{G}>\mathrm{C}$ & & & $1(2.0)$ & - & - & - \\
\hline Codon 13 & - & - & - & $1(2.0)$ & - & $1(1.0)$ \\
\hline Codon $1337 \mathrm{G}>\mathrm{A}$ & $1(2.3)$ & - & - & - & $1(1.0)$ & - \\
\hline Codon 13 38G $>\mathrm{C}$ & $1(2.3)$ & - & - & - & - & - \\
\hline Codon $1338 \mathrm{G}>\mathrm{A}$ & - & - & $2(3.9)$ & - & $1(1.0)$ & - \\
\hline Codon 61 & - & - & - & - & - & - \\
\hline Codon 61 182A $>\mathrm{T}$ & - & - & $2(3.9)$ & - & - & - \\
\hline Wild type & $39(88.6)$ & $42(95.5)$ & $42(82.4)$ & $48(94.1)$ & $92(89.3)$ & $97(94.2)$ \\
\hline Indeterminate & $2(4.5)$ & $2(4.5)$ & - & - & - & $1(1.0)$ \\
\hline Total & 44 & 44 & 51 & 51 & 103 & 103 \\
\hline Not Measured & 59 & 59 & 52 & 52 & 0 & 0 \\
\hline
\end{tabular}

Data are presented as $\mathrm{n}(\%)$.

Statistical analyses. Demographic data are presented as a mean $\pm \mathrm{SD}$ or $\mathrm{n}(\%)$. Agreement between PANAMutyper ${ }^{\mathrm{TM}}$ and PNAClamp ${ }^{\mathrm{TM}}$ for tissues, cell blocks, or effusion samples was determined based on overall agreement and Cohen's kappa value. McNemar's test was used to identify any discordance between the two methods for tissues, cell blocks, and effusion samples, after categorizing all patients into: i) wild and ii) mutant type.

Diagnostic performance of the two methods for detecting KRAS mutations in pleural fluids was presented with sensitivity, specificity, positive predictive value, and negative predictive value, with the mutation status in all samples combined as a reference standard. A "wild type" in the reference standard was defined as a failure to detect any mutation in all samples combined, identified using either PANAMutyper ${ }^{\mathrm{TM}}$ or PNAClamp ${ }^{\mathrm{TM}}$. In addition, Cohen's kappa statistic was calculated to compare the agreement of each method between the result of pleural fluid and the reference standard.

A two-sided $p$-Value of equal or less than 0.05 was considered statistically significant. All statistical analyses were performed using the SAS 9.4 software (SAS Institute, Inc., Cary, NC, USA).

\section{Results}

Patient characteristics. Baseline demographic characteristics of enrolled patients are summarized in Table I. Of 103 patients, $65(63.1 \%)$ were males. The mean age of all patients was $72 \pm 12$ years. Fifty-one $(49.5 \%)$ patients had a history of smoking. Eighty-nine patients were diagnosed as primary lung cancer (72 patients with NSCLC and 17 patients with SCLC). Fourteen patients had other malignancies ( 3 with stomach cancer, 2 with mesothelioma, 2 with ovarian cancer, 2 with thyroid cancer, 1 with breast cancer, 1 with endometrial cancer, 1 with esophageal cancer, 1 with head and neck cancer, and 1 with lymphoma).
Among these 103 subjects, primary malignancies were diagnosed based on pathologic confirmation of the primary tumor $(n=81)$ or by examination of pleural specimens only (pleural tissue or cell block of the pleural fluid) without confirmation of the primary tumor site $(n=22)$. Pleural malignancy was diagnosed via pleural biopsy $(n=10)$, cell block or cytology of the pleural fluid $(n=62)$, or exclusion of other causes of pleural effusion $(n=31)$. The major histological type of lung cancer was adenocarcinoma (54.4\%).

Comparison of KRAS mutations detected by PANAMutyper ${ }^{T M}$ and PNAClamp ${ }^{T M}$. KRAS mutations were detected in 18 $(17.5 \%)$ of 103 patients (Table II). Among these 18 patients with a KRAS mutation, 11 were males and 9 had a history of smoking. There was no KRAS mutation in patients with squamous cell carcinoma or small cell carcinoma of the lung. All 18 patients with KRAS mutations were detected by PANAMutyper ${ }^{\mathrm{TM}}$ using any sample type, but only 7 cases were detected by PNAClamp ${ }^{\mathrm{TM}}$.

Codon 12 had the highest incidence of KRAS mutations, present in 11 patients ( 4 with codon $1235 \mathrm{G}>\mathrm{T}$, 3 with codon $1235 \mathrm{G}>\mathrm{A}, 2$ with codon $1234 \mathrm{G}>\mathrm{A}, 1$ with codon 12 $34 \mathrm{G}>\mathrm{C}$, and 1 with codon $1235 \mathrm{G}>\mathrm{C}$ ), followed by codon 13 present in 5 patients ( 2 with codon $1337 \mathrm{G}>\mathrm{A}, 2$ with codon $1338 \mathrm{G}>\mathrm{A}$, and 1 with codon $1338 \mathrm{G}>\mathrm{C}$ ) and codon 61 in 2 patients $(182 \mathrm{~A}>\mathrm{T})$.

Concordance of PANAMutyper ${ }^{T M}$ and PNAClamp ${ }^{T M}$. Detailed comparisons of KRAS mutations according to sample type and detection method are shown in Tables II and 
Table IV. Concordance between PANAMutyper ${ }^{T M}$ and PNAClamp ${ }^{T M}$ for the detection of KRAS mutations.

\begin{tabular}{|c|c|c|c|c|c|c|c|}
\hline & \multicolumn{7}{|c|}{ PNAClamp ${ }^{\mathrm{TM}}$} \\
\hline & \multicolumn{2}{|c|}{ Mutation } & \multirow{2}{*}{$\begin{array}{c}\text { Wild type/ } \\
\text { indeterminate }\end{array}$} & \multirow[t]{2}{*}{ Total } & \multirow{2}{*}{$\begin{array}{l}\text { K coefficient } \\
(95 \% \mathrm{CI})^{*}\end{array}$} & \multirow{2}{*}{$\begin{array}{l}\text { Overall agreement* } \\
\qquad(95 \% \mathrm{CI})\end{array}$} & \multirow{2}{*}{$\begin{array}{c}\text { McNemar's test } \\
p \text {-Value* }\end{array}$} \\
\hline & Codon 12 & Codon 13 & & & & & \\
\hline \multicolumn{8}{|l|}{ PANAMutyper ${ }^{\mathrm{TM}}$} \\
\hline \multicolumn{8}{|l|}{ Tissue } \\
\hline \multicolumn{8}{|l|}{ Mutation } \\
\hline Codon 12 35G $>\mathrm{T}$ & 0 & 0 & 1 & 1 & 0 & $0.93(0.81-0.99)$ & 0.0833 \\
\hline Codon 13 37G $>$ A & 0 & 0 & 1 & 1 & & & \\
\hline Codon 13 38G $>\mathrm{C}$ & 0 & 0 & 1 & 1 & & & \\
\hline Wild type/Indeterminate & 0 & 0 & 41 & 41 & & & \\
\hline Total & 0 & 0 & 44 & 44 & & & \\
\hline \multicolumn{8}{|l|}{ Cell block } \\
\hline \multicolumn{8}{|l|}{ Mutation } \\
\hline Codon $1234 \mathrm{G}>\mathrm{C}$ & 1 & 0 & 0 & 1 & $0.45(0.11-0.80)$ & $0.88(0.76-0.96)$ & 0.0143 \\
\hline Codon $1235 \mathrm{G}>\mathrm{T}$ & 1 & 0 & 1 & 2 & & & \\
\hline Codon 12 35G $>\mathrm{A}$ & 0 & 0 & 1 & 1 & & & \\
\hline Codon 12 35G $>C$ & 0 & 0 & 1 & 1 & & & \\
\hline Codon $1338 \mathrm{G}>\mathrm{A}$ & 0 & 1 & 1 & 2 & & & \\
\hline Codon $61182 \mathrm{~A}>\mathrm{T}$ & 0 & 0 & 2 & 2 & & & \\
\hline Wild type/indeterminate & 0 & 0 & 42 & 42 & & & \\
\hline Total & 2 & 1 & 48 & 51 & & & \\
\hline \multicolumn{8}{|l|}{ Effusion } \\
\hline \multicolumn{8}{|l|}{ Mutation } \\
\hline Codon $1234 \mathrm{G}>\mathrm{C}$ & 1 & 0 & 0 & 1 & $0.60(0.31-0.88)$ & $0.94(0.88-0.98)$ & 0.0143 \\
\hline Codon 12 34G $>$ A & 0 & 0 & 2 & 2 & & & \\
\hline Codon $1235 \mathrm{G}>\mathrm{T}$ & 2 & 0 & 1 & 3 & & & \\
\hline Codon $1235 \mathrm{G}>\mathrm{A}$ & 1 & 0 & 2 & 3 & & & \\
\hline Codon $1337 \mathrm{G}>\mathrm{A}$ & 0 & 0 & 1 & 1 & & & \\
\hline Codon $1338 \mathrm{G}>\mathrm{A}$ & 0 & 1 & 0 & 1 & & & \\
\hline Wild type/indeterminate & 0 & 0 & 92 & 92 & & & \\
\hline Total & 4 & 1 & 98 & 103 & & & \\
\hline
\end{tabular}

Data are presented as n. *All patients were categorized into two groups: mutant and wild type.

III. In tumor tissues, KRAS mutations were only identified in $3(6.8 \%)$ out of 44 patients by using PANAMutyper ${ }^{\mathrm{TM}}$ (Table III). In cell blocks,9 $(17.6 \%)$ and $3(5.9 \%)$ out of 51 patients were found to have KRAS mutations using PANAMutyper ${ }^{\mathrm{TM}}$ and PNAClamp ${ }^{\mathrm{TM}}$, respectively. The three mutations identified using PNAClamp ${ }^{\mathrm{TM}}$ were concordant with those detected using PANAMutyper ${ }^{\mathrm{TM}}$. The six other KRAS mutations detected by PANAMutyper ${ }^{\mathrm{TM}}$ were identified as wild type by PNAClamp ${ }^{\mathrm{TM}}$.

Regarding pleural effusion samples, PANAMutyper ${ }^{\mathrm{TM}}$ identified KRAS mutations in $11(10.7 \%)$ samples, whereas PNAClamp $^{\mathrm{TM}}$ identified KRAS mutations in $5(4.9 \%)$ samples. The 5 mutations identified by PNAClamp ${ }^{\mathrm{TM}}$ were consistent with those detected by PANAMutyper ${ }^{\mathrm{TM}}$. The 6 other KRAS mutations detected by PANAMutyper ${ }^{\mathrm{TM}}$ were identified as wild type by PNAClamp ${ }^{\mathrm{TM}}$.

The diagnostic concordance between PANAMutyper ${ }^{\mathrm{TM}}$ and PNAClamp ${ }^{\mathrm{TM}}$ in tissues, cell blocks, and effusion samples is shown in Table IV. K coefficients between the two methods were 0.45 and 0.60 for cell blocks and effusion samples, respectively, indicating a moderate agreement between PANAMutyper ${ }^{\mathrm{TM}}$ and PNAClamp ${ }^{\mathrm{TM}}$. However, McNemar's test showed a significant superiority of PANAMutyper ${ }^{\mathrm{TM}}$ over PNAClamp ${ }^{\mathrm{TM}}$ in both cell blocks and pleural effusion samples.

Diagnostic performance of pleural effusion to detect KRAS mutations. The diagnostic performance of pleural effusion compared to results obtained from all samples combined, showed a sensitivity of $61 \%$, a specificity of $100 \%$, a positive predictive value of $100 \%$, and a negative predictive value of $92 \%$ for PANAMutyper ${ }^{\mathrm{TM}}$. For PNAClamp ${ }^{\mathrm{TM}}$, the diagnostic performance of pleural effusion showed a sensitivity of $28 \%$, a specificity of $100 \%$, a positive predictive value of $100 \%$, and a negative predictive value of $87 \%$ (Table V). 
in vivo $33:$ 945-954 (2019)

Table V. Diagnostic performance of two mutation detection methods using pleural effusion samples compared to results obtained from all samples combined.

\begin{tabular}{|c|c|c|c|c|c|c|c|c|}
\hline \multirow[t]{2}{*}{ Effusion } & \multicolumn{2}{|c|}{$\begin{array}{c}\text { Results from all } \\
\text { samples combined }\end{array}$} & \multirow[t]{2}{*}{ Total } & \multirow[t]{2}{*}{$\begin{array}{l}\text { Sensitivity } \\
(95 \% \mathrm{CI})^{*}\end{array}$} & \multirow[t]{2}{*}{$\begin{array}{l}\text { Specificity } \\
(95 \% \mathrm{CI})\end{array}$} & \multirow[t]{2}{*}{$\begin{array}{c}\text { PPV } \\
(95 \% \mathrm{CI})\end{array}$} & \multirow[t]{2}{*}{$\begin{array}{c}\text { NPV } \\
(95 \% \mathrm{CI})\end{array}$} & \multirow[t]{2}{*}{$\begin{array}{l}\text { K coefficient } \\
(95 \% \mathrm{CI})\end{array}$} \\
\hline & Mutation & Wild type & & & & & & \\
\hline \multicolumn{9}{|c|}{ PANAMutyper ${ }^{\mathrm{TM}}$} \\
\hline Mutation & 11 & 0 & 11 & $0.61(0.36-0.83)$ & $1.00(0.96-1.00)$ & $1.00(0.72-1.00)$ & $0.92(0.85-0.97)$ & $0.72(0.53-0.91)$ \\
\hline Wild type & 7 & 85 & 92 & & & & & \\
\hline \multicolumn{9}{|c|}{ PNAClamp $^{\mathrm{TM} \dagger}$} \\
\hline Mutation & 5 & 0 & 5 & $0.28(0.10-0.53)$ & $1.00(0.96-1.00)$ & $1.00(0.48-1.00)$ & $0.87(0.78-0.93)$ & $0.39(0.14-0.63)$ \\
\hline Wild type & 13 & 84 & 97 & & & & & \\
\hline
\end{tabular}

${ }^{*} p$-Value of 0.025 in McNemar's test for comparing sensitivities. ${ }^{\dagger}$ A total of 102 out of 103 samples were evaluated due to one indeterminate result. 95\% Cl: 95\% Confidence Interval.

\section{Discussion}

The aim of the present study was to compare the performance of PANAMutyper ${ }^{\mathrm{TM}}$ and PNAClamp ${ }^{\mathrm{TM}}$ for the detection of KRAS mutations in matched tumor tissues, cell blocks, and pleural effusion samples. In addition, the diagnostic performance of both methods using pleural fluid samples for the detection of KRAS mutations was investigated.

The detection rate of KRAS mutations varied from $0 \%$ to $17.6 \%$, depending on specimen and detection method. There was an acceptable concordance between PANAMutyper ${ }^{\mathrm{TM}}$ and PNAClamp ${ }^{\mathrm{TM}}$ for cell blocks and pleural effusion samples. However, tissue specimens did not show any agreement due to their low sensitivities for detecting KRAS mutations. PANAMutyper ${ }^{\mathrm{TM}}$ had a superior diagnostic performance over PNAClamp ${ }^{\mathrm{TM}}$ for cell blocks and pleural effusion specimens. Compared to results obtained for all samples combined, a good diagnostic performance regarding the detection of KRAS mutations was achieved when pleural effusion samples were used. In the current study, rates of KRAS mutations detected by PANAMutyper ${ }^{\mathrm{TM}}$ in tissue, cell block, and pleural effusion samples were 6.8\%, 17.6\%, and $10.7 \%$, respectively. These rates detected by PNAClamp ${ }^{\mathrm{TM}}$ were $0 \%, 5.9 \%$, and $4.9 \%$, respectively.

The frequency of KRAS mutations is known to vary among different ethnic groups. In Caucasian patients, the prevalence of KRAS mutations in lung cancer is $17 \%$ in the COSMIC database (16) and 33\% in lung adenocarcinoma in TCGA (17). In Eastern Asians, the prevalence of KRAS mutations is 2.3$9.4 \%(18,19)$. In the present study, the frequency of KRAS mutations caused by lung cancer from all specimens combined was $16.9 \%$ (15 out of 89 ) and $4.5 \%$ (4 out of 89 ) detected by PANAMutyper ${ }^{\mathrm{TM}}$ and PNAClamp ${ }^{\mathrm{TM}}$, respectively.

Mutation of the RAS gene usually occurs in adenocarcinoma (20) and rarely in squamous cell carcinoma, while it never occurs in small cell lung cancer (21). Wu et al. have studied the prevalence of KRAS mutations in Taiwanese and found that the prevalence is $3.8 \%$ (9 out of 237 patients) in lung cancer (18). Among these 9 patients with KRAS mutations, $8(88.9 \%)$ had adenocarcinoma while one $(11.1 \%)$ had squamous cell carcinoma (18). The results of the present study correspond well with the previous study on the Taiwanese. Thirteen $(86.7 \%)$ of 15 patients with KRAS mutations exhibited adenocarcinoma histology in the present study. However, squamous cell carcinoma or small cell carcinoma identified no KRAS mutations.

Approximately $97 \%$ of KRAS mutations in non-small cell lung cancer occur in exons 2 and 3 . They are commonly found in codon 12 , occasionally in codon 13 and rarely in codon $61(20,22,23)$. In the current study, among the 18 detected KRAS mutations, most of them were detected in codon 12 (11 patients), followed by those in codon 13 (5 patients) and codon 61 (2 patients).

Specific subtypes of KRAS mutations could be associated with different clinical implications, such as drug sensitivity or prognosis. Regarding the distinct subtype of KRAS mutations, the most common mutation is a codon $1234 \mathrm{G}>\mathrm{T}$ point mutation, followed by codon $1235 \mathrm{G}>\mathrm{T}$ and codon 12 $35 \mathrm{G}>\mathrm{A}$ mutations (24). The present study also demonstrated that four KRAS mutations had a codon $1235 \mathrm{G}>\mathrm{T}$ point mutation, while three KRAS mutations had a codon 12 $35 \mathrm{G}>\mathrm{A}$ point mutation. The Taiwanese study has indicated that the most commonly identified KRAS mutation is codon $1235 \mathrm{G}>\mathrm{T}$, followed by codon $1235 \mathrm{G}>\mathrm{A}$, consistent with our results. These findings suggest that specific subtype of KRAS mutations could be different depending on ethnic group. Further large-scale studies are needed to verify the clinical role of the distinct subtypes of KRAS mutations.

Direct sequencing of DNA is traditionally a reasonable approach to identify the KRAS mutation status. We have previously reported that the diagnostic performance of PNA clamping is better than that of direct sequencing (9), 
however, more sensitive methods are being developed these days. Superior results of the PANAMutyper ${ }^{\mathrm{TM}}$ technology for detecting EGFR mutation using specimens of plasma and bronchoalveolar lavage fluid have been reported recently $(15,25)$. To the best of our knowledge, this is the first study concerning the diagnostic performance of PNA clampingassisted fluorescence melting curve analysis, by comparing matched tissue and cell block specimens with pleural effusion samples for the detection of KRAS mutations.

Our study has several limitations. First, the number of patients was not large. Second, the number of tissue and cell block specimens was not matched to that of pleural effusion samples because some specimens were of insufficient volume following routine pathological examination.

Taken together, we found that PANAMutyper ${ }^{\mathrm{TM}}$ had a superior diagnostic performance over PNAClamp ${ }^{\mathrm{TM}}$ for the detection of KRAS mutations, although there was concordance between the PANAMutyper ${ }^{\mathrm{TM}}$ and PNAClamp ${ }^{\mathrm{TM}}$ results. Furthermore, the good diagnostic accuracy of using pleural fluid sample can provide useful clinical information offering better prediction and personalized therapy.

\section{Clinical Practice Points}

- PNAClamp ${ }^{\mathrm{TM}}$ is currently used to detect KRAS mutations because of its superior diagnostic performance over conventional Sanger sequencing.

- PANAMutyper ${ }^{\mathrm{TM}}$ is a novel technology that integrates PNAClamp $^{\mathrm{TM}}$ and PANA S-Melting ${ }^{\mathrm{TM}}$.

- PANAMutyper ${ }^{\mathrm{TM}}$ and PNAClamp ${ }^{\mathrm{TM}}$ were compared for the detection of KRAS mutations in patients with malignant pleural effusion.

- PANAMutyper ${ }^{\mathrm{TM}}$ had a diagnostic superiority for the detection of KRAS mutations compared to PNAClamp ${ }^{\mathrm{TM}}$.

- Frequency and specific subtypes of KRAS mutations in the current study correspond well with those in a previous study on Taiwanese.

- PANAMutyper ${ }^{\mathrm{TM}}$ can be used for more sensitive and accurate detection of KRAS mutations.

\section{Conflicts of Interest}

The Authors declare no conflicts of interest.

\section{Authors' Contributions}

Conception and design: Su Yeon Choi, Seung Joon Kim; Development of methodology: Hyung Woo Kim, Sang Hoon Jeon, Bit Na Kim, Nahyeon Kang; Acquisition of data: Chang Dong Yeo, Chan Kwon Park; Analysis and interpretation of data (e.g., statistical analysis, biostatistics, computational analysis): Mi Sun Park, Hyeon Woo Yim; Writing, review, and/or revision of the manuscript: $\mathrm{Su}$ Yeon Choi, Jong Y. Park, Seung Joon Kim; Administrative, technical, or material support: Yoon Ho Lee, Kyo Young Lee, Young Kyoon Kim, Sug Hyung Lee; Study supervision: Seung Joon Kim.

\section{Acknowledgements}

This work was supported by a grant (2014R1A2A1A11052422) from the National Research Foundation (NRF) funded by the Korean government (MSIP). Statistical consultation was supported by a grant (HI14C1062) from the Korea Health Technology R\&D Project through the Korean Health Industry Development Institute (KHIDI), which is funded by the Ministry of Health \& Welfare, Republic of Korea.

\section{References}

1 Simanshu DK, Nissley DV and McCormick F: RAS proteins and their regulators in human disease. Cell 170: 17-33, 2017. PMID: 28666118. DOI: 10.1016/j.cell.2017.06.009

2 Min YJ: Realities of KRAS-mutated non-small cell lung cancer. Korean J Intern Med 32: 442, 2017. PMID: 28490719. DOI: 10.3904/kjim.2017.148

3 Ying M, Zhu XX, Zhao Y, Li DH and Chen LH: KRAS Mutation as a biomarker for survival in patients with non-small cell lung cancer, a meta-analysis of 12 randomized trials. Asian Pac J Cancer Prev 16: 4439-4445, 2015. PMID: 26028111. DOI: 10.7314/APJCP.2015.16.10.4439

4 Haigis KM: KRAS Alleles: The devil is in the detail. Trends Cancer 3: 686-697, 2017. PMID: 28958387. DOI: 10.1016/ j.trecan.2017.08.006

5 Lee SH, Jo EJ, Eom JS, Mok JH, Kim MH, Lee K, Kim KU, Park HK, Lee CH, Kim YD and Lee MK: Predictors of recurrence after curative resection in patients with early-stage non-small cell lung cancer. Tuberc Respir Dis (Seoul) 78: 341348, 2015. PMID: 26508921. DOI: 10.4046/trd.2015.78.4.341

6 Park S, Kim JY, Lee SH, Suh B, Keam B, Kim TM, Kim DW and Heo DS: KRAS G12C mutation as a poor prognostic marker of pemetrexed treatment in non-small cell lung cancer. Korean J Intern Med 32: 514-522, 2017. PMID: 28407465. DOI: 10.3904/kjim.2015.299

7 Paik SS, Hwang IK, Park MJ and Lee SH: Pemetrexed continuation maintenance versus conventional platinum-based doublet chemotherapy in EGFR-negative lung adenocarcinoma: retrospective analysis. Tuberc Respir Dis (Seoul) 81: 148-155, 2018. PMID: 29527843. DOI: 10.4046/trd.2017.0090

8 Yeo CD, Kim JW, Kim KH, Ha JH, Rhee CK, Kim SJ, Kim YK, Park CK, Lee SH, Park MS and Yim HW: Detection and comparison of EGFR mutations in matched tumor tissues, cell blocks, pleural effusions, and sera from patients with NSCLC with malignant pleural effusion, by PNA clamping and direct sequencing. Lung Cancer 81: 207-212, 2013. PMID: 23726527. DOI: $10.1016 /$ j.lungcan.2013.04.023

9 Kang JY, Park CK, Yeo CD, Lee HY, Rhee CK, Kim SJ, Kim SC, Kim YK, Park MS and Yim HW: Comparison of PNA clamping and direct sequencing for detecting KRAS mutations in matched tumour tissue, cell block, pleural effusion and serum from patients with malignant pleural effusion. Respirology 20 : 138-146, 2015. PMID: 25302858. DOI: 10.1111/resp.12413

10 Lee KY, Kim HJ, Kim SJ, Yoo GH, Kim WD, Oh SY and Kim WS: PNA-mediated PCR clamping for the detection of EGFR mutations in non-small cell lung cancer. Tuberc Respir Dis 69: 271-278, 2010. DOI: $10.4046 / \operatorname{trd} .2010 .69 .4 .271$

11 Han HS, Lim SN, An JY, Lee KM, Choe KH, Lee KH, Kim ST, Son SM, Choi SY, Lee HC and Lee OJ: Detection of EGFR mutation status in lung adenocarcinoma specimens with different 
proportions of tumor cells using two methods of differential sensitivity. J Thorac Oncol 7: 355-364, 2012. PMID: 22157369. DOI: $10.1097 /$ JTO.0b013e31823c4c1b

12 Kim HJ, Lee KY, Kim YC, Kim KS, Lee SY, Jang TW, Lee MK, Shin KC, Lee GH, Lee JC, Lee JE and Kim SY: Detection and comparison of peptide nucleic acid-mediated real-time polymerase chain reaction clamping and direct gene sequencing for epidermal growth factor receptor mutations in patients with non-small cell lung cancer. Lung Cancer 75: 321-325, 2012. PMID: 21930325. DOI: 10.1016/j.lungcan.2011.08.005

13 Kim HR, Lee SY, Hyun DS, Lee MK, Lee HK, Choi CM, Yang SH, Kim YC, Lee YC, Kim SY, Jang SH, Lee JC and Lee KY: Detection of EGFR mutations in circulating free DNA by PNAmediated PCR clamping. J Exp Clin Cancer Res 32: 50, 2013. PMID: 23927790. DOI: 10.1186/1756-9966-32-50

14 Jeon SH, Kim HW, Kim BN, Kang N, Yeo CD, Park CK, Kim YK, Lee YH, Kim TJ, Lee KY, Lee SH, Park JY, Park MS, Yim HW and Kim SJ: Comparison of PNA clamping-assisted fluorescence melting curve analysis and PNA clamping in detecting EGFR mutations in matched tumor tissue, cell block, pleural effusion and blood of lung cancer patients with malignant pleural effusion. In Vivo 33: 595-603, 2019. PMID: 30804147. DOI: 10.21873 /invivo.11516

15 Han JY, Choi JJ, Kim JY, Han YL and Lee GK: PNA clampingassisted fluorescence melting curve analysis for detecting EGFR and KRAS mutations in the circulating tumor DNA of patients with advanced non-small cell lung cancer. BMC Cancer 16: 627, 2016. PMID: 27519791. DOI: 10.1186/s12885-016-2678-2

16 Prior IA, Lewis PD and Mattos C: A comprehensive survey of Ras mutations in cancer. Cancer Res 72: 2457-2467, 2012. PMID: 22589270. DOI: 10.1158/0008-5472.CAN-11-2612

17 The Cancer Genome Atlas Research Network: Comprehensive molecular profiling of lung adenocarcinoma. Nature 511: 543550, 2014. PMID: 25079552. DOI: 10.1038/nature 13385

$18 \mathrm{Wu}$ CC, Hsu HY, Liu HP, Chang JW, Chen YT, Hsieh WY, Hsieh JJ, Hsieh MS, Chen YR and Huang SF: Reversed mutation rates of KRAS and EGFR genes in adenocarcinoma of the lung in Taiwan and their implications. Cancer 113: 31993208, 2008. PMID: 18932251. DOI: 10.1002/cncr.23925

19 Smits AJ, Kummer JA, Hinrichs JW, Herder GJ, ScheidelJacobse KC, Jiwa NM, Ruijter TE, Nooijen PT, LooijenSalamon MG, Ligtenberg MJ, Thunnissen FB, Heideman DA, de Weger RA and Vink A: EGFR and KRAS mutations in lung carcinomas in the Dutch population: increased EGFR mutation frequency in malignant pleural effusion of lung adenocarcinoma. Cell Oncol (Dordr) 35: 189-196, 2012. PMID: 22528563. DOI: 10.1007/s13402-012-0078-4
20 Rodenhuis S, van de Wetering ML, Mooi WJ, Evers SG, van Zandwijk N and Bos JL: Mutational activation of the K-ras oncogene. A possible pathogenetic factor in adenocarcinoma of the lung. N Engl J Med 317: 929-935, 1987. PMID: 3041218. DOI: $10.1056 /$ NEJM198710083171504

21 Mitsudomi T, Viallet J, Mulshine JL, Linnoila RI, Minna JD and Gazdar AF: Mutations of ras genes distinguish a subset of nonsmall-cell lung cancer cell lines from small-cell lung cancer cell lines. Oncogene 6: 1353-1362, 1991. PMID: 1679529. DOI: 10.1016/0169-5002(92)90122-Z

22 Suda K, Tomizawa K and Mitsudomi T: Biological and clinical significance of KRAS mutations in lung cancer: an oncogenic driver that contrasts with EGFR mutation. Cancer Metastasis Rev 29: 49-60, 2010. PMID: 20108024. DOI: 10.1007/s10555010-9209-4

23 Matikas A, Mistriotis D, Georgoulias V and Kotsakis A: Targeting KRAS mutated non-small cell lung cancer: A history of failures and a future of hope for a diverse entity. Crit Rev Oncol Hematol 110: 1-12, 2017. PMID: 28109399. DOI: 10.1016/j.critrevonc.2016.12.005

24 Wood K, Hensing T, Malik R and Salgia R: Prognostic and Predictive Value in KRAS in Non-Small-Cell Lung Cancer: A Review. JAMA Oncol 2: 805-812, 2016. PMID: 27100819. DOI: 10.1001/jamaoncol.2016.0405

25 Park S, Hur JY, Lee KY, Lee JC, Rho JK, Shin SH and Choi CM: Assessment of EGFR mutation status using cell-free DNA from bronchoalveolar lavage fluid. Clin Chem Lab Med, 2017. PMID: 28195541. DOI: 10.1515/cclm-2016-0302 